\title{
Crucial electronic contributions to measures of surface diffusion by He atom scattering
}

\author{
Guido Fratesi, ${ }^{1,2}$ Gil Alexandrowicz, ${ }^{3}$ Mario Italo Trioni, ${ }^{2}$ Gian Paolo Brivio,,${ }^{1,2}$ and William Allison ${ }^{3}$ \\ ${ }^{1}$ Dipartimento di Scienza dei Materiali, Università di Milano-Bicocca, Via Cozzi 53, 20125 Milano, Italy \\ ${ }^{2}$ ETSF and CNISM, UdR Milano-Bicocca, Via Cozzi 53, 20125 Milano, Italy \\ ${ }^{3}$ Cavendish Laboratory, University of Cambridge, Madingley Road, Cambridge CB3 OHE, United Kingdom
}

(Received 5 May 2008; published 27 June 2008)

\begin{abstract}
We examine theoretically recent $\mathrm{He}$ atom scattering measurements of $\mathrm{Na} / \mathrm{Cu}(001)$, identifying a nontrivial relation between the dynamics of the electron distribution and that of the Na ions. The magnitude of the calculated variations in the charge density, and their dependence on the local density of adsorbates, account for the correlated three-dimensional (3D) motion experimentally observed. The results of this study further highlight the sensitivity of helium scattering to the electron distribution of the sample and point out the role of electronic contributions in high-resolution measures of surface dynamics.
\end{abstract}

DOI: 10.1103/PhysRevB.77.235444

PACS number(s): 68.49.Bc, 68.43.Jk, 73.20.-r, 79.20.Rf

\section{INTRODUCTION}

Surface dynamics plays a critical role in many fundamental processes such as crystal growth, molecular selfassembly, and heterogeneous catalysis. The study of the dynamics is interesting also from a fundamental point of view, since it supplies unique insight into the atomic scale interactions at surfaces. In this respect, He atom scattering (HAS) is a particularly useful method to investigate structural and dynamical properties of a surface, thanks to the inertness of helium and to its negligible penetration depth. In fact the analysis of He scattering cross sections significantly advanced our understanding of important topics such as surface corrugations, ${ }^{1}$ surface phonons, ${ }^{2}$ and adsorbate diffusion. ${ }^{3} \mathrm{~A}$ major improvement in the field is represented by the recently developed ${ }^{3} \mathrm{He}$ spin-echo (HSE) spectrometer, ${ }^{4,5}$ which is capable of measuring surface motion on a time scale previously inaccessible to experiment. ${ }^{6}$

HAS is in some sense complementary to scanning tunneling microscopy (STM). These techniques have been widely used to study ultraslow (STM) (Ref. 7) and ultrafast (HAS) surface diffusion processes. Since the measuring process involves electrons rather than nuclei, a common feature of these two experiments is that the position and motion of atoms or molecules at a surface is inferred indirectly: through the electron distribution at the sample surface, which provides the scattering potential for He in HAS, and through the tunneling current between the sample and the tip in STM. Many studies of surface structure have shown that the relation between the measured corrugation and the position of the atoms on the surface can be complex. ${ }^{8,9}$ While this indirect relation requires particular attention when interpreting the measurements, at the same time it enriches the potentials of these methods to focus on electronic states, especially when refined theoretical modeling is combined to experiments. In contrast to structural studies, surface diffusion measurements are typically analyzed assuming that the electron distribution and the tunneling current simply follow the position of the surface atoms.

The study presented here illustrates that electronic properties can play a fundamental role in the measurement of surface dynamics by He scattering. We analyze theoretically
$\mathrm{Na} / \mathrm{Cu}(001)$. Recent HSE measurements of this system ${ }^{10}$ have addressed the previously unexplained coverage dependence of Na surface diffusion ${ }^{11,12}$ and have identified a new component of motion oriented perpendicular to the surface. Such perpendicular motion could be detected from a Na coverage of 0.04 ML, and its effect rapidly increased with deposition of adatoms. Time scales and temperature dependence are comparable with those of the lateral hopping process of $\mathrm{Na}$ atoms between surface sites. Furthermore, numerical models in which the height of a $\mathrm{Na}$ atom changes within a $0.2 \AA$ range, depending on the local adsorbate configuration, reproduced the experimental observations. ${ }^{10}$ However, the physical origins of the perpendicular motion remained unclear and are the subject of the present investigation. At fixed coverage, thermal fluctuations in the adsorbate density give a variety of adsorbate configurations, each with a different local concentration. We have, therefore, studied both nuclear and electronic contributions to the apparent height of a $\mathrm{Na}$ atom on $\mathrm{Cu}(001)$ as a function of the local concentration of neighboring $\mathrm{Na}$ adatoms while keeping the coverage constant. The theoretical procedure, based on ab initio structural optimizations and the effective medium theory for the $\mathrm{He}$ surface interaction, is described in Sec. II. In Sec. III we first evaluate the role of the motion of $\mathrm{Na}$ ions, and then we estimate the contribution due to alterations of the electron distribution at the surface. Finally, in Sec. IV a comparison of our results with experimental findings is presented, followed by the conclusions.

\section{THEORETICAL METHODS}

All structures and electron distributions presented in this paper have been obtained by ab initio simulations in the framework of density-functional theory (DFT) (Refs. 13 and 14), adopting the Perdew-Burke-Ernzerhof ${ }^{15}$ generalized gradient approximation for the exchange and correlation functional. Periodically repeated slabs have been chosen as a model for the surface. Dipole field corrections were adopted to keep periodicity in the electrostatic potential without spurious electric fields inside the slab, as described by Bengtsson. ${ }^{16}$ Slabs consisting of three layers only provide accurately enough coordinates and energies upon $\mathrm{Na}$ adsorp- 
(a)

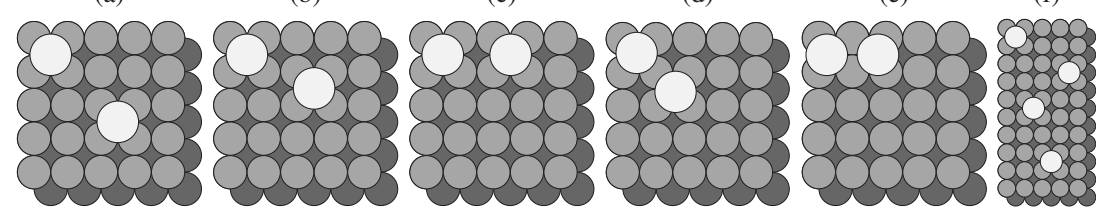

FIG. 1. (a)-(e) Top view of the possible configurations of two $\mathrm{Na}$ adatoms (bright circles) in a $\mathrm{Cu}(001)(5 \times 5)$ cell. (f) Example of $(5 \times 10)$ unit cell with four adatoms. tion. The bottom two layers were kept fixed at the bulktruncated positions, while the upper one was allowed to relax. Na atoms were placed on this side of the slab and relaxed together with the upper layer. The plane-wave ultrasoft pseudopotential method ${ }^{17}$ was used as implemented in the PWSCF code of the Quantum-ESPRESSO distribution. ${ }^{18}$

The He scattering process is described by determining the classical turning point (CTP). In the case of elastic specular reflection, He atoms are scattered by the surface at the distance $z^{\mathrm{CTP}}$ at which the atom-surface potential equals their incoming kinetic energy $E_{\text {kin }}$ projected along the normal to the surface. The interaction of a noble gas at a surface is currently beyond the realm of DFT owing to approximations in the exchange-correlation functional. Therefore, we shall rely on estimates instead of direct calculations. We have chosen to adopt the effective medium theory (EMT) (Ref. 19): we approximate the repulsive part of the interaction at a given point as the energy of $\mathrm{He}$ in a uniform medium with the same electron density, $\rho$, as that protruding from the surface at the same point. According to Esbjerg and Nørskov, ${ }^{20}$ the leading term is proportional to $\rho$, with proportionality constant $\alpha=45 \mathrm{eV} \AA^{3}$ (Ref. 21), and therefore depends exponentially on the He-surface distance. It is not necessary here to consider the attractive part-a damped van der Waals interaction yielding a small potential well, $6 \mathrm{meV}$ for $\mathrm{He} /$ $\mathrm{Cu}(001)$ (Ref. 22)—since our results do not depend critically on fine details of the interaction potential, as will be shown below. Taking $E_{\mathrm{kin}}=10 \mathrm{meV}$ and $135^{\circ}$ for the scattering angle as in the experiments, the reflection condition is given by $\rho(x, y, z)=1.9 \times 10^{-4} \AA^{-3} \equiv \rho^{\mathrm{EMT}}$. This equation defines implicitly the function $z^{\mathrm{CTP}}(x, y)$, representing the apparent height probed by $\mathrm{He}$ atoms. In particular, $z^{\mathrm{CTP}}\left(x_{0}, y_{0}\right)$ is our estimate for the height of an adatom with nucleus in $\left(x_{0}, y_{0}, z_{0}\right)$. This is a local maximum of the $z^{\mathrm{CTP}}$ function and it amounts to $\approx 3 \AA$ above the adatom nucleus.

\section{RESULTS}

We first estimate the contribution to the perpendicular motion given directly by the dynamics of $\mathrm{Na}$ nuclei. The coverage investigated experimentally is $0.08 \mathrm{ML}$ at most, which is well within the low-coverage regime. ${ }^{23}$ From our calculations, the height of the nucleus of a $\mathrm{Na}$ atom in the equilibrium position (hollow site), as measured from the first unperturbed $\mathrm{Cu}$ layer, is $2.35 \AA$. This value increases by 0.09 and $0.15 \AA$ for atoms sitting in bridge and top positions, respectively. It is important to notice that the Na atoms do not spend enough time in these sites to show up in the measurement. ${ }^{10}$ Therefore, it is possible to relate the observation of perpendicular motion with the height of $\mathrm{Na}$ nuclei only if the equilibrium adsorption coordinate is modified. This modification could be due to the interactions with the other adsorbates when the local concentration of $\mathrm{Na}$ atoms changes (notice that the coverage is constant during the measuring process, so no alteration of the bond length with coverage would be detected by HAS, neither is one expected ${ }^{24}$ ). To evaluate this hypothesis, we have performed a set of DFT simulations at fixed coverage $(0.08 \mathrm{ML})$ for a model where two $\mathrm{Na}$ atoms are adsorbed in a periodically repeated (5 $\times 5) \mathrm{Cu}(001)$ cell. The adatoms were initially put in hollow sites at different distances, and coordinates were optimized, resulting in the structures [Figs. 1(a)-1(e)] displayed in the corresponding panels of Fig. 1. The Na height coincides for configurations [Figs. 1(a)-1(d)] within $0.01 \AA$. In the limiting case [Fig. $1(\mathrm{e})]$, the atoms are intentionally placed very close to each other, giving a total energy that is $200 \mathrm{meV}$ larger than that in the case of Fig. 1(a). However, also for this configuration the nuclear coordinate is modified by a tiny amount, only $0.03 \AA$ higher than that in the other cases, the small difference originating from the repulsive interaction pushing $\mathrm{Na}$ atoms toward the bridge site. An atomistic interpretation of the perpendicular motion is therefore excluded.

To estimate the contribution to the observed height of a $\mathrm{Na}$ atom arising from possible alterations in the electron density, consider again the five test cases illustrated in Figs. 1(a)-1(e); the corresponding valence electron density at points on top of one of the two equivalent $\mathrm{Na}$ atoms is plotted in Fig. 2 as a function of the height above the $\mathrm{Cu}$ nuclei in the first layer, $z$. The peak at $\approx 2.3 \AA$, which follows the tiny displacement of the nucleus, is mostly due to $2 s 2 p$ elec-

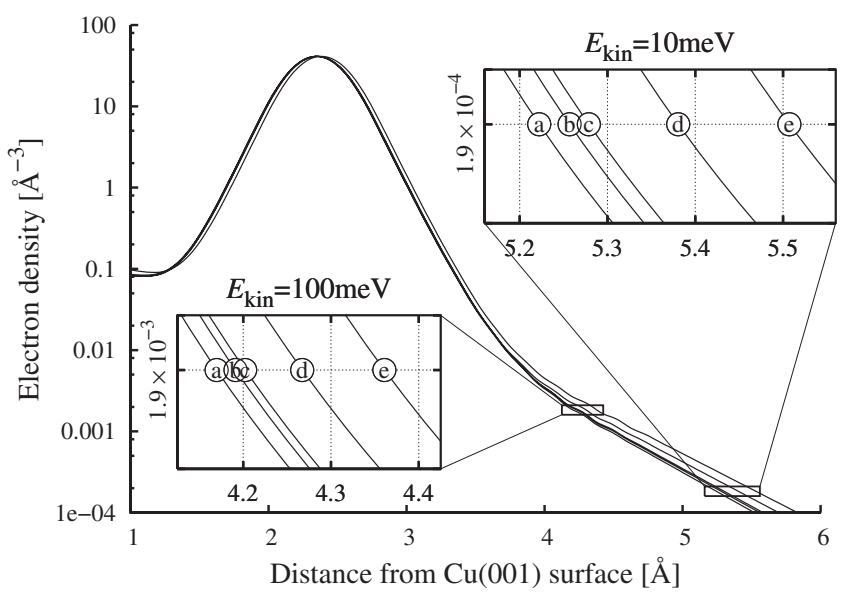

FIG. 2. Valence electron density at points on top of a $\mathrm{Na}$ nucleus, located $\approx 2.3 \AA$ above the surface. Lines marked (a)-(e) refer to corresponding configurations shown in Fig. 1. Densities between $10^{-4}$ and $10^{-3} \AA^{-3}$ represent the results of an ideal calculation with an infinite number of basis functions extracted by data exponential fit over the same range. Insets magnify the values of the classical turning point for beam kinetic energies of 10 and $100 \mathrm{meV}$. 
trons; for $\rho$ smaller than $10^{-3} \AA^{-3}(z>4 \AA)$ the contribution stemming from the partially filled $3 s$ level becomes dominant and determines the turning point for the kinetic energy used in the experiments. In this range, $\rho\left(x_{0}, y_{0}, z\right)$ is well described by an exponential decay. Insets magnify the values of $z^{\mathrm{CTP}}$. At a kinetic energy of $10 \mathrm{meV}$ (upper inset), the apparent height of $\mathrm{Na}$ is $5.2 \AA$ above the surface $\mathrm{Cu}$ atoms for the configuration of Fig. 1(a) and increases systematically up to $5.5 \AA$ when the distance between the atoms is reduced [Figs. 1(b)-1(e)]. As a consequence, atoms with a closer $\mathrm{Na}$ neighbor will appear higher than those with a farther one. This effect has the same order of magnitude as the experimental one and is an order of magnitude larger than the corresponding variation of the nuclear coordinate $z_{0}$. At larger He kinetic energies the spread in the values of $z^{\mathrm{CTP}}$ is smaller but can still be much larger than that of nuclear coordinates. This is shown in the lower inset of Fig. 2 for $E_{\text {kin }}=100 \mathrm{meV}$ (about $50 \%$ the effect reported at $10 \mathrm{meV}$ ). The robustness of our qualitative results with respect to $E_{\text {kin }}$ also proves that fine details in the estimate of the He-surface interaction are not essential. Hence, additional contributions (van der Waals, nonlocality, and hybridization energy ${ }^{25}$ ) can be neglected in the present treatment.

The systematic increase in the classical turning point upon approaching another $\mathrm{Na}$ adatom, shown in Fig. 2, is also present in more general cases where the local concentration of adatoms increases. To show this effect, possible $\mathrm{Na}$ arrangements occurring at the surface can be modeled by larger supercells with many adatoms. However, this is limited by the computational requirements to perform numerical simulations. It is helpful to notice that the accuracy of $z^{\mathrm{CTP}}$ trends does not depend strongly on the kinetic-energy cutoff for expanding wave functions. ${ }^{26}$ In practice, we could reduce its value from 27 to $20 \mathrm{Ry}$ and keep the difference in $z^{\mathrm{CTP}}$ among the various configurations within $10 \%$ of the converged result, which is accurate enough for our analysis. This allowed us to perform additional DFT calculations for (5 $\times 10)$ supercells with different arrangements of two (0.04 $\mathrm{ML})$ or four (0.08 ML) Na atoms, as shown for example in Fig. 1(f). In all these simulations, the $\mathrm{Cu}$ surface was kept frozen to the unperturbed coordinates and $\mathrm{Na}$ atoms were put in hollow sites at equal height to best isolate the electronic contribution. The apparent height $z^{\mathrm{CTP}}$ corresponding to $E_{\text {kin }}=10 \mathrm{meV}$ was estimated as before.

The results of these calculations can be analyzed by using a suitable measure for the local concentration. For convenience, we choose the same measure as in Ref. 10, namely, the dipole-dipole interaction energy per atom, $E_{i}^{d-d}$. We remark that this choice does not imply a particular force law. For the adatom $i$, one has

$$
E_{i}^{d-d}=\frac{1}{2} \sum_{j}^{\prime} \frac{2 \mu_{\mathrm{av}}^{2}}{\left|\mathbf{R}_{j}-\mathbf{R}_{i}\right|^{3}} .
$$

Here $i$ and $j$ is the label adatoms with nuclei in $\mathbf{R}_{i}$ and $\mathbf{R}_{j}$, the summation ranges over all $\mathrm{Na}$ atoms $j$ different than $i$ (in models based on the supercell approach, periodic replicas are also to be included). $\mu_{\mathrm{av}}$ is the average dipole of $\mathrm{Na}$ adatoms, the factor 2 accounts for interaction with image charges. Har-

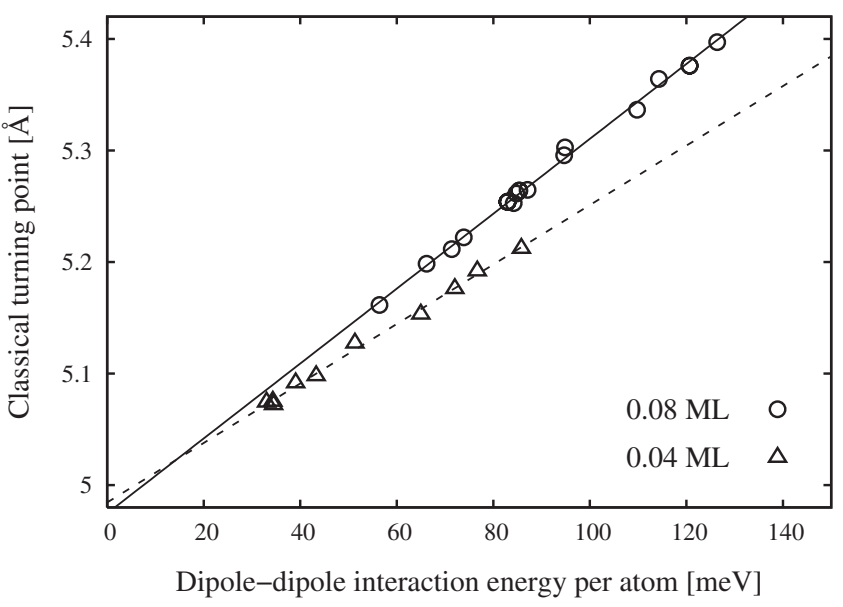

FIG. 3. Estimate of the classical turning point for He kinetic energy of $10 \mathrm{meV}$ on top of $\mathrm{Na}$ atoms as function of corresponding dipole-dipole interaction energy per atom, defined in Eq. (1). The solid and dashed lines are linear fits through the data [see Eqs. (2) and (3)].

tree atomic units are used in Eq. (1) and in the following, unless otherwise specified. Overall, $\mathrm{Na}$ atoms with 26 different values of the $E^{d-d}$ parameter were considered, sampling cases with local concentrations higher and lower than the average one. For each of them, $z^{\text {CTP }}$ is plotted in Fig. 3 against the corresponding value of $E^{d-d}$. Notice the monotonic increase of $z^{\mathrm{CTP}}$ with $E^{d-d}$. Therefore, atoms at larger local concentrations appear higher on the surface to the incoming He beam due to the more extended electron density. Moreover, $z^{\mathrm{CTP}}$ follows within $0.005 \AA$ a linear dependence on $E^{d-d}$, at both coverages considered:

$$
\begin{array}{ll}
z^{\mathrm{CTP}}=3.36 \AA / \mathrm{eV} \quad E^{d-d}+\text { const } & (0.08 \mathrm{ML}), \\
z^{\mathrm{CTP}}=2.66 \AA / \mathrm{eV} E^{d-d}+\text { const } & (0.04 \mathrm{ML}) .
\end{array}
$$

Many other quantities could have been adopted in order to parametrize the local concentration, but generally such a simple functional dependence would not result.

We now show by simple arguments that the dependence of the classical turning point on the local $\mathrm{Na}$ concentration arises from changes in the potential acting on the electrons, $V$. Let $\mathbf{R}_{0}=\left(x_{0}, y_{0}, z_{0}\right)$ be the coordinate of a Na nucleus and let us focus again on points $\left(x_{0}, y_{0}, z\right)$ above it, for values of $z \approx z^{\text {CTP }}$. At low Na coverage, the dominant contribution to $V$ by other $\mathrm{Na}$ atoms is the electrostatic potential, $V^{d}$, related to their electric dipole. By assuming $z-z_{0}$ smaller than the $\mathrm{Na}-\mathrm{Na}$ separation, $V^{d}$ is given by;

$$
V^{d}\left(x_{0}, y_{0}, z\right) \approx \sum_{j \neq 0} \frac{2 \mu_{\mathrm{av}}\left(z-z_{0}\right)}{\left|\mathbf{R}_{j}-\mathbf{R}_{0}\right|^{3}}=\frac{2\left(z-z_{0}\right)}{\mu_{\mathrm{av}}} E_{0}^{d-d} .
$$

As a consequence, $V$ is proportional to $E_{0}^{d-d}$, with the ratio $2\left(z^{\mathrm{CTP}}-z_{0}\right) / \mu_{\mathrm{av}}$. Next, we recall that the spill out of the electron cloud into the vacuum is well described by an exponential function. Hence, within the Wentzel-Kramers-Brillouin approximation, the electron density can be written as $\rho\left(x_{0}, y_{0}, z\right) \approx \rho_{0} e^{-2 k\left(z-z_{0}\right)}$, where $k=\sqrt{2(V-\varepsilon)}(\varepsilon$ is the average energy of the states contributing to $\rho$ ). By combining these 
expressions, we estimate the variation in the turning point with the local concentration as $\frac{\partial z^{\mathrm{CTP}}}{\partial E^{d-d}}=\frac{2\left(z^{\mathrm{CTP}}-z_{0}\right)^{2}}{\mu_{\mathrm{av}} k^{2}}$. At $0.08 \mathrm{ML}$, such a rate amounts to $2.67 \AA / \mathrm{eV}$, in reasonable agreement with the value obtained by fitting DFT results [see Eq. (2)]. The above formula reproduces the decrease in the height dependence on concentration when the coverage is reduced (1.74 $\AA / \mathrm{eV}$ at $0.04 \mathrm{ML})$ or when the He kinetic energy is increased (1.07 $\AA / \mathrm{eV}$ at $100 \mathrm{meV})$, as described above. This argument suggests that the phenomenon should be observed in other systems, particularly when a strong dipole is created in the adsorption. At present, however, the number of published experiments is too small to make any firm conclusion.

\section{DISCUSSION AND CONCLUSIONS}

The relation we established between the $\mathrm{Na}$ apparent height and the local concentration allowed us to interpret the experimental findings. Surface diffusion causes the local concentration around a given adatom to fluctuate with time, producing variations in the apparent height measured by HAS. As a result, the perpendicular motion will be characterized by the same time scale and activation energy as the lateral hopping process, as observed in the experiment. Additional insight is given by the probability distribution of the apparent heights of $\mathrm{Na}$ adatoms, which we have computed by means of molecular dynamics (MD) simulations ( $T$ $=155 \mathrm{~K}$; simulation parameters as in Ref. 10), through the evaluation of the parameter $E^{d-d}$ and Eqs. (2) and (3). Results are reported in Fig. 4. At 0.08 ML, such distribution is fairly broad, displaying a full width at half maximum (FWHM) of $0.14 \AA$, comparable with the experimentally observed extent of the perpendicular motion. At a lower coverage, the probability distribution gets significantly narrower (at $0.04 \mathrm{ML}$ the FWHM is $0.07 \AA$ ), indicating a reduction in the perpendicular motion, which follows the trend of experimental observation.

In conclusion, we have shown that the previously unexplained perpendicular motion observed in experiment is purely an electronic effect, arising from the alterations of the electron distribution outside the surface, which follow the

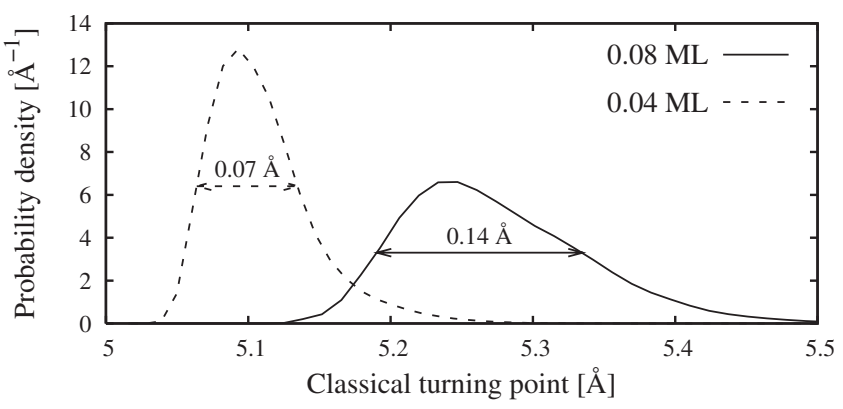

FIG. 4. Probability distribution of the classical turning point determined by molecular dynamics simulations by using Eqs. (2) and (3).

fluctuations with time of the local concentration of adatoms. Such small alterations are probed by HSE to an extent that was estimated by taking the classical turning point in the EMT. The calculated range of the apparent motion, tenths of angstroms, is consistent with the measurements and is one order of magnitude larger than the change in the height of the $\mathrm{Na}$ nuclei. The decrease in the perpendicular motion at lower $\mathrm{Na}$ coverage is also reproduced.

Our investigation shows from first principles how correlated adsorbate motion gives rise to nontrivial electronic rearrangements, which do not simply follow the position of the ions. Such electronic contributions are appreciable in HAS measures of surface diffusion and, under particular circumstances, are significantly larger than the ionic ones. Such a sensitivity, coupled to advanced theoretical models, could exploit in the future the unique properties of HAS in studies primarily addressing electronic properties.

\section{ACKNOWLEDGMENTS}

This work was supported by the EU Network of Excellence NANOQUANTA (Grant No. NMP4-CT-2004-500198) and by the MIUR of Italy (Grant No. 2005021433-003). Computational resources were made available by CINECA through INFM grants. We acknowledge the useful discussions with G. Scoles and J. Ellis.
${ }^{1}$ D. Farías and K.-H. Rieder, Rep. Prog. Phys. 61, 1575 (1998).

${ }^{2}$ G. Benedek and J. P. Toennies, Surf. Sci. 299-300, 587 (1994).

${ }^{3}$ F. Hofmann and J. P. Toennies, Chem. Rev. (Washington, D.C.) 96, 1307 (1996).

${ }^{4}$ A. P. Jardine, S. Dworski, P. Fouquet, G. Alexandrowicz, D. J. Riley, G. Y. H. Lee, J. Ellis, and W. Allison, Science 304, 1790 (2004).

${ }^{5}$ P. Fouquet, A. P. Jardine, S. Dworski, G. Alexandrowicz, W. Allison, and J. Ellis, Rev. Sci. Instrum. 76, 053109 (2005).

${ }^{6} \mathrm{G}$. Alexandrowicz and A. P. Jardine, J. Phys.: Condens. Matter 19, 305001 (2007).

${ }^{7}$ E. Ganz, S. K. Theiss, I.-S. Hwang, and J. Golovchenko, Phys. Rev. Lett. 68, 1567 (1992).

${ }^{8}$ K. H. Rieder, G. Parschau, and B. Burg, Phys. Rev. Lett. 71,
1059 (1993).

${ }^{9}$ W. A. Hofer, A. S. Foster, and A. L. Shluger, Rev. Mod. Phys. 75, 1287 (2003).

${ }^{10} \mathrm{G}$. Alexandrowicz, A. P. Jardine, H. Hedgeland, W. Allison, and J. Ellis, Phys. Rev. Lett. 97, 156103 (2006).

${ }^{11}$ A. Cucchetti and S. C. Ying, Phys. Rev. B 60, 11110 (1999).

${ }^{12}$ J. Ellis, A. P. Graham, F. Hofmann, and J. P. Toennies, Phys. Rev. B 63, 195408 (2001).

${ }^{13}$ P. Hohenberg and W. Kohn, Phys. Rev. 136, B864 (1964).

${ }^{14}$ W. Kohn and L. J. Sham, Phys. Rev. 140, A1133 (1965).

${ }^{15}$ J. P. Perdew, K. Burke, and M. Ernzerhof, Phys. Rev. Lett. 77, 3865 (1996).

${ }^{16}$ L. Bengtsson, Phys. Rev. B 59, 12301 (1999).

${ }^{17}$ D. Vanderbilt, Phys. Rev. B 41, 7892 (1990). 
${ }^{18}$ S. Baroni, A. Dal Corso, S. de Gironcoli, and P. Giannozzi (http://www.pwscf.org).

${ }^{19}$ J. K. Nørskov and N. D. Lang, Phys. Rev. B 21, 2131 (1980).

${ }^{20}$ N. Esbjerg and J. K. Nørskov, Phys. Rev. Lett. 45, 807 (1980).

${ }^{21}$ M. Manninen, J. K. Nørskov, M. J. Puska, and C. Umrigar, Phys. Rev. B 29, 2314 (1984).

${ }^{22}$ A. P. Graham, D. Fang, E. M. McCash, and W. Allison, Phys. Rev. B 57, 13158 (1998).

${ }^{23}$ A. P. Graham and J. P. Toennies, Phys. Rev. B 56, 15378 (1997).

${ }^{24}$ R. D. Diehl and R. McGrath, J. Phys.: Condens. Matter 9, 951 (1997).
${ }^{25}$ J. F. Annett and R. Haydock, Phys. Rev. B 34, 6860 (1986).

${ }^{26}$ This is possible thanks to the following considerations: (i) We shall be interested here in variations of $z^{\mathrm{CTP}}$ rather than values; (ii) The turning point depends on the electron density far from the atomic cores, and is not much affected by the accuracy of the wave functions close to nuclei; (iii) Structural optimization of the system (for which the pseudopotentials used would require a cutoff of $27 \mathrm{Ry}$ ) is not needed, since we have shown that the adsorption height does not vary significantly. The charge density was always expanded up to $216 \mathrm{Ry}$. 